\title{
Zero-current-switching pulse charger for high capacity batteries in renewable energy applications
}

\author{
Sunil G. Abeyratne*, Nirmana Perera, Hiranya Jayakody, Kosala Samarakoon and Rukshan Bulathge \\ Department of Electrical and Electronic Engineering, Faculty of Engineering, University of Peradeniya, Peradeniya.
}

Revised: 09 October 2015; Accepted: 21 January 2016

\begin{abstract}
Fast charging of high capacity batteries is of great concern, especially in renewable energy and electric vehicle (EV) applications. Pulse charging has become the preferred method for fast charging with heavy currents. However, it presents drawbacks due to switching losses involved in hardswitching of semiconductor devices. This paper presents a soft switched pulse charging mechanism for high capacity $\mathrm{Pb}$-acid and $\mathrm{Li}$-ion batteries, which minimises switching losses that makes the methodology very suitable for implementing the National Electric Code (NEC) level-1, level-2 chargers or specially the level-3 charger more conveniently for EVs. The fully clamped quasi-resonant DC link (FCQDL) converter generates current pulses to charge the battery in a zero-current switching (ZCS) manner to minimise switching losses. The simulated and experimental results show that the proposed charger can implement constant current (CC) and constant voltage $(\mathrm{CV})$ modes of charging. The experimental results show that the proposed charging methodology reduces the charging time of $\mathrm{Pb}$-acid batteries by $14.01 \%$ in contrast to the conventional $\mathrm{CC}-\mathrm{CV}$ charging method. Furthermore, discharging curves for the conventional and pulse charging methods are compared to evaluate the effective useful charge that is put into the battery. The results reveal that the effective charge capacity of a battery is significantly improved due to the use of pulse charging.
\end{abstract}

Keywords: Current pulse charging, fast charging, high capacity battery chargers, renewable energy, zero-current-switching.

\section{INTRODUCTION}

High capacity battery storage systems are widely used in renewable energy applications and electric vehicles (Lachs \& Sutanto, 1992; Horie et al., 2008; Garimella \& Nair, 2009; Etxeberria \& Vechiu, 2010). Large scale energy storage helps to store the electricity produced by renewable sources, and therefore meet the energy demand of the grid whilst adding flexibility to the grid (Lachs \& Sutanto, 1992).

At present, several types of batteries such as $\mathrm{Pb}$-acid, $\mathrm{NiMH}$ and Li-ion are used in large scale energy storage applications (Horie et al., 2008; Zhang et al., 2011), including hybrid and fully electric vehicles. High capacity $\mathrm{Pb}$-acid batteries are low in energy density but are used in many renewable energy applications owing to its reliability and low cost (Horie et al., 2008). Apart from wind and solar applications, $\mathrm{Pb}$-acid batteries are used in houses connected to smart grids (Garimella \& Nair, 2009). However, Li-ion batteries dominate the prevailing energy storage field, particularly in the electric vehicle industry where the high energy density factor plays a critical role in favouring the vehicle dynamics by reducing the weight of the battery pack used (Horie et al., 2008; Zhang et al., 2011). Additionally, Li-ion batteries are currently integrated with wind turbines and solar PVs as a storage device in countries around the world.

Regardless of the battery type, a fast and efficient charging mechanism to charge the batteries integrated to renewable energy applications is needed for the effective capturing of energy from intermittent energy resources (Barton \& Infield, 2004). Fast charging offers a significant advantage when it comes to electric vehicle industry where the charging time poses a considerable effect on the market of the vehicle.

Generally, both $\mathrm{Pb}$-acid and Li-ion batteries are charged using the constant current-constant voltage (CC-CV) charging methodology (Asadi et al., 2011). In 
the constant current (CC) phase, a constant current stream is fed to the battery till the rated voltage of the battery is reached. However, the use of continuous current charging circuits involving semiconductor devices operated in the active region cause an active device loss during the charging process. Evidently, this loss is considerably higher for large scale batteries and consequently has a negative impact on the efficiency of the charging circuit. Furthermore, in the search for reduced charging times, if the charging current exceeds the recommended level of the battery, undesirable chemical reactions take place inside the battery causing the battery to heat up, and ultimately shortening its lifespan (Belov \& Yang, 2008; Horie et al., 2008; Methekar \& Ramadesigan, 2010). As such, implementing fast charging mechanisms for high capacity batteries, whilst maintaining the efficiency is a prevailing problem due to several reasons.

The preferred method of fast charging Li-ion and $\mathrm{Pb}$-acid batteries uses a current pulse stream in the CC charging phase, instead of a constant-continuous current to charge the battery (Li et al., 2001). In order to generate the required current pulses, a topology which involves continuous switching of power semiconductor devices should be employed (Mohan et al., 2003). Most of the traditional pulse-charging methods use hard switching to facilitate this requirement. Apparently, this is disadvantageous due to the substantial losses involved in hard switching with large currents, especially when operated at higher frequencies (Mi \& Bai, 2011). Note that it is essential to use higher switching frequencies to reduce the circuit size (Mohan et al., 2003). The losses may be ignored for small voltage applications; however, they are significant for the applications using high current and voltage ratings (Mohan et al., 2003; Mi \& Bai, 2011) such as automotive and renewable energy applications.

This switching loss has a negative impact on charging large scale batteries from renewable energy sources, where the limited energy output of the source needs to be fully utilised. For instance, an electric vehicle charger with a low efficiency would cause a considerable amount of electrical energy loss, which may have been produced using fossil fuels such as coal, petrol, diesel, etc. Therefore, when it comes to the final carbon credit calculation for an electric vehicle (Anderson \& Anderson, 2010), the anticipated advantage over a conventional vehicle may not be achieved. Hence, wasting a huge amount of energy to minimise the charging time of an electric vehicle challenges the purpose of replacing conventional vehicles with EVs (Anderson \& Anderson, 2010).

\section{Effect of pulse charging on batteries}

Recent studies have shown that using current pulse streams as opposed to continuous current to charge high capacity Li-ion batteries not only reduces the charging time, but also improves the lifespan of the battery ( $\mathrm{Li}$ et al., 2001; Chen, 2009; Methekar \& Ramadesigan, 2010). The underline reasons identified for these types of observations are addressed as follows.

Pulse charging provides adequate time (between the current pulses) for the battery to stabilise its chemical reactions before recommencing the charging process. Consequently, chemical reactions inside the battery are able to keep in pace with the energy input while reducing the unwanted chemical reactions, which could take place inside the battery, thus increasing the power transfer rate (Methekar \& Ramadesigan, 2010). Furthermore, negative effects caused due to concentration polarisation in the battery are also avoided (Li et al., 2001). Evidently, these factors advantageously reduce the time consumed for constant voltage charging phase by a considerable amount (Li et al., 2001), thus decreasing the overall charging time. For instance, assume that a Li-ion battery is charged at a rate of $1 \mathrm{C}$ (Kutkut et al., 1998) using the conventional CC-CV method. A Li-ion battery of the same capacity can be charged using a current pulse stream maintaining the average of the current pulses to be at $1 \mathrm{C}$ during the charging process, while improving the charging time by $14 \%$ (Chen, 2009). Moreover, the active material utilisation inside the battery due to pulse charging leads to better discharge capacity and longer battery life (Li et al., 2001; Lin \& Yen, 2008).

Not only Li-ion batteries, but Pb-acid batteries also yield similar characteristics under current pulse charging. The experiments carried out by this research group show that the use of current pulse charging to charge $\mathrm{Pb}$-acid batteries improves the charging time as well as the discharging capacity (Abeyratne \& Aydemir, 1994; Anderson \& Anderson, 2010). Preliminary experiments conducted during this research showed that pulse charging improves the charging time of a $\mathrm{Pb}$-acid battery by $14 \%$ and the effective charge retaining capacity by $5.9 \%$.

Although pulse charging provides promising results, it holds a primary drawback lying with its practical implementation with regard to high capacity battery banks. As mentioned earlier, it is apparent that hard switching of the semiconductor devices to derive the required current pulse stream causes a considerable 
amount of switching losses. The existing fast charging methodologies hold this problem.

In Ke et al. (2007), Chen (2009), and Chen (2011) different approaches which utilise pulse charging for Li-ion and Pb-acid batteries are presented. Ke et al. (2007) employed a buck zero-current switching PWM converter to implement pulse charging on a $\mathrm{Pb}$-acid battery. However, the charger does not have the capability to change the magnitude of the current pulses fed to the battery making it difficult to implement the CC-CV charging topology. Furthermore, proper current and voltage feedback from the battery needs to be taken into consideration in order to properly maintain the current value in the $\mathrm{CC}$ phase and the voltage value in the CV phase. On the other hand, the circuit assumes a DC voltage input, which could change depending on the energy source connected to the charger.

A duty-varied voltage pulse charger for Li-ion batteries was developed by Chen (2009). This methodology further enhances the performance of current pulse charging by varying the duty ratio of the current pulse stream supplied to the battery. However, the proposed charger utilises hard switching, causing switching losses as a result. Although this may not create a huge effect for small scale batteries, it would be a concern in charging high capacity batteries. Chen (2011) used a buck converter topology with zero-current switching as a battery charger. However, the final current waveform passing through the battery is not pulsed and therefore, the charging time is degraded.

This study aimed at eliminating the aforementioned limitations of the existing chargers and developing a feasible and robust high-capacity battery charger, utilising a fully clamped quasi-resonant DC link (FCQDL) converter. The proposed methodology implements a fast charging mechanism for high capacity batteries while significantly minimising the switching losses. The switching losses are minimised through a FCQDL converter (Abeyratne \& Aydemir, 1994), which enables zero-current switching (Hua \& Lee, 1995). This permits fast charging while maintaining a higher efficiency. In addition, a control topology was developed to maintain precise CC-CV operation. It is observed that charging time can be further reduced by varying the frequency of the current pulse stream depending on the battery type. The charger can be connected to renewable energy sources as well as to electric vehicles or hybrids to keep the state of charge at the prescribed limits efficiently.
In addition to a previous study by the authors (Abeyratne et al., 2012), this paper presents a comprehensive analysis on the operation of the charger at each stage. Furthermore, a prototype of the charger has been developed and experimental results were analysed.

\section{METHODOLOGY}

In the formulated methodology, a current pulse stream was employed to charge the battery in both the $\mathrm{CC}$ and CV phases. The FCQDL converter (Abeyratne \& Aydemir, 1994) implemented in this topology produces the required current pulse stream through a zero-current switching mechanism (Hua \& Lee, 1995). A current pulse stream of a pre-defined magnitude and pulse width is first supplied to the battery until the battery terminal voltage reaches its rated value (i.e. CC phase). Then the charger switches to the $\mathrm{CV}$ phase. In this phase, the battery terminal voltage is kept at its rated value by controlling the pulse heights of the output current pulse stream of the FCQDL converter (Abeyratne \& Aydemir, 1994; Abeyratne et al., 2012).

It should be emphasised that the proposed battery charger uses the same current pulse charging topology for both $\mathrm{CC}$ and $\mathrm{CV}$ phases, thus removing the need for an additional circuitry for the $\mathrm{CV}$ phase. In the $\mathrm{CV}$ phase, a voltage feedback loop from the battery allows the control of the magnitude of the current pulses in the stream to reduce appropriately, as the battery reaches its fully-charged level. The charging process ceases when the average current input to the battery reaches $2 \%$ of its rated $\mathrm{C}$ value as recommended.

The design of the proposed $\mathrm{Li}$-ion/ $\mathrm{Pb}$-acid battery charger consists of three major sub circuits; the boost converter to obtain the required DC voltage (Mohan et al., 2003), the FCQDL converter to obtain the fast charging pulse mechanism (Abeyratne \& Aydemir, 1994), and the control unit to handle the charging process.

The power flow and the functionalities of each of these sub-circuits are discussed in the following sections.

\section{Power topology of the charger}

The conceptual power topology of the charger can be described as follows. The energy source, which supplies the required power to the charger depends upon the application for which the charger is used for. For example, if the charger is used to store the energy produced by an array of solar panels, the energy source 
block is effectively the output of the solar panel array. On the other hand, when the charger is utilised to charge the high capacity battery in an electric vehicle, the energy source would be a $110-230$ V AC outlet. An AC energy source would require a diode bridge to rectify the voltage, whereas a DC source can be directly connected to the boost converter input.

The functionality of the boost converter is conventional in the sense of operation, and the topology provides almost a unity power factor condition (Mohan et al., 2003; Muthuramalingam \& Himavathi, 2009; Kuperman et al., 2011). The voltage feedback control loop implemented with the boost converter ensures that the voltage at the boost converter output is maintained as required by the battery (Muthuramalingam \& Himavathi, 2009; Kuperman et al., 2011). The control topology is discussed in detail in the following sections.

The output of the boost converter is connected to a thyristor bridge, which acts as a controlled voltage source to the FCQDL converter. The need for the thyristor bridge is discussed later. Furthermore, it is possible to replace the thyristor bridge by an H-bridge isolation unit joined with a diode-bridge unit if isolation is required (Mohan et al., 2003; Mi et al., 2008). In either case, the input to the FCQDL converter can be rendered to a form of a controlled voltage source (Abeyratne \& Aydemir, 1994).

The FCQDL converter takes input power from the controlled voltage source and executes a path-switching process which generates a periodic stream of current pulses (with zero-current switching) at its output. This current pulse stream is utilised to charge the high capacity battery unit.

The functionalities of each of these sub-circuits are discussed as follows.

\section{Principle of operation of the pulse charger}

1) Fully clamped quasi-resonant DC link converter: This is the core unit that delivers the principle operation of the pulse charging concept. Its input is the output of the thyristor bridge, and the output is connected to the high capacity $\mathrm{Pb}$-acid/ Li-ion battery.

As shown in Figure 1, this DC-DC topology consists of four insulated gate bipolar transistor (IGBT) switches, which are switched in a strategic predetermined order to generate the current pulse stream. The inductor $L_{0}$ (comparatively smaller in size compared to $L_{d}$ ) and the capacitor $C_{0}$ form part of the resonant tank, which facilitates the task of soft switching the DC link converter IGBTs (Abeyratne \& Aydemir, 1994; Hua \& Lee, 1995).

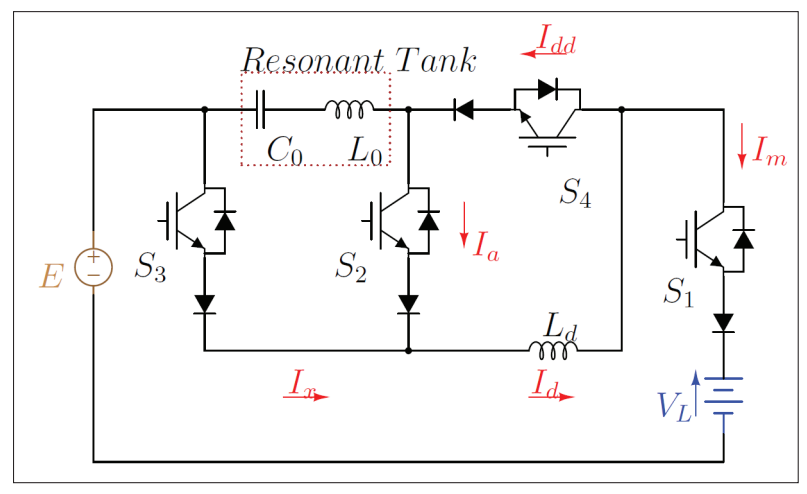

Figure 1: Fully clamped quasi-resonant DC-link (FCQDL) converter

The current handling capability of the topology depends upon the inductor $L_{d}$, which acts as the current source. The current through this inductor, $I_{d}$, can be kept approximately equal to a DC reference current level due to the high-frequency switching in the DC link converter.

Consider the voltage across $L_{d}$ to be,

$$
V_{L_{d}}=L_{d} \frac{d I_{d}}{d t}
$$

Therefore the change current $I_{d}$ is,

$$
d I_{d}=\frac{V_{L_{d}}}{L_{d}} d t
$$

Here, $V_{L_{d}}$ represents the voltage across the inductor $L_{d}$. It is apparent that due to the high-frequency switching operation, $d t$ is much small, hence the change in $I_{d}$ is not appreciable. In fact, for a few switching cycles it can be assumed that $I_{d}$ remains constant.

The magnitude of $I_{d}$ relates to the voltage difference between the input of the DC link converter, $V_{e}$, and the battery terminal voltage, $V_{b}$, as,

$$
I_{d}=k_{I_{d}}\left(V_{e}-V_{b}\right)
$$

where, $k_{I_{d}}$ is a positive constant.

According to equation (3), once the charging process commences, the battery terminal voltage $\left(V_{b}\right)$ starts to increase, reducing the magnitude of $I_{d}$. In order to avoid this behaviour, $V_{b}$ is sensed and $V_{e}$ is changed accordingly by the embedded control scheme to maintain $I_{d}$ at the desired level (Abeyratne \& Aydemir, 1994; Jiang \& Dougal, 2004).

The four IGBTs in the DC link converter employ a repetitive sequence of zero-current switching operations to guide the constant DC link current $\left(I_{d}\right)$ through the 
battery for a specified amount of time $\left(t_{m}\right)$ within a single switching cycle of the whole converter, which spans across a time interval $T_{p}$. Note that the current through the inductor $L_{d}$ is $I_{d}$ throughout the duration of $T_{p}$. The zero-current switching sequence of the IGBTs is illustrated in Figure 2. As a result, the battery is subjected to a current pulse stream with a duty of $t_{m} / T_{p}$. More importantly, due to the zero current, switching losses are avoided.

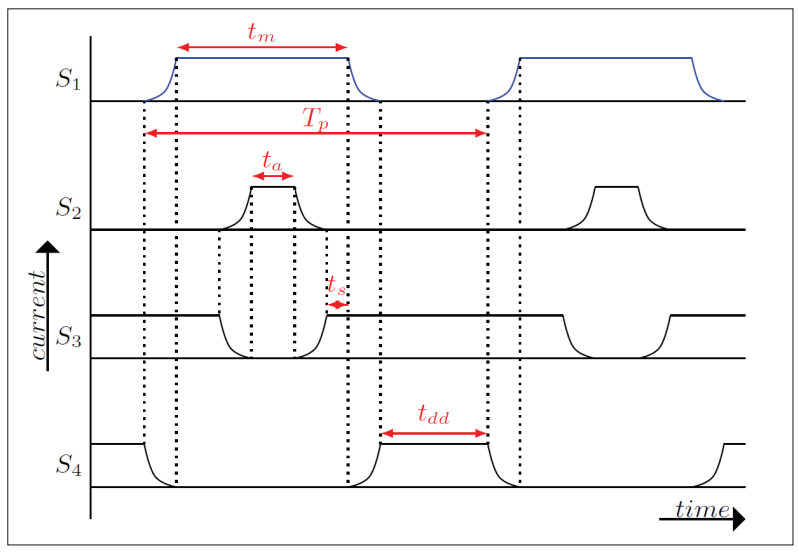

Figure 2: Switching diagram for the four switches during one switching cycle of the FCQDL converter

The width of the current pulse (i.e. the value of time $t_{m}$ ) is governed by the individual switching times of the four IGBT switches in the DC link topology. Evidently, the frequency of the output current pulse stream, $f_{p}=1 / T_{p}$ depends on the switching frequency of the overall converter.

The switching sequence, which enables zero-current switching can be explained using four unique modes of operation of the DC link converter. In order to simplify the explanation of the circuit, assume that IGBTs denoted by $S_{1}, S_{3}$ and $S_{4}$ (refer to Figure 1) are initially switched on.

Mode 1

The equivalent circuit for Mode 1 is depicted in Figure 3(a)(I). Applying Kirchhoff's current law to the circuit yields,

$$
I_{S_{3}}=I_{d}=I_{S_{1}}+I_{S_{4}}
$$

Here $I_{S_{1}}$ and $I_{S_{4}}$ represent the current through switches $S_{1}$ and $S_{4}$, respectively. As $S_{1}$ is turned on, since the battery is also a voltage source, the voltage held off by $S_{4}$ is matched by inductor $L_{0}$ satisfying the voltage equation of the loop. The result is a heavily exited resonant tank comprising $C_{0}$ and $L_{0}$. Consequently, the value of $I_{S_{4}}$ decreases in a resonant manner, forcing $I_{S_{1}}$ to increase in order to satisfy equation (4). At the end of the transient, $I_{S_{1}}\left(=I_{m}\right)$ becomes equal to $I_{d}$, and this directly charges the battery. The duty cycle of the current pulse stream fed to the battery is basically decided by the amount of time the circuit operates in Mode 1. Due to the fact that $I_{S_{4}}=0$ now, $S_{4}$ can be switched off safely without any switching loss.

Mode 2

When the appropriate current pulse width for charging is reached, IGBT $S_{2}$ is turned on as shown in Figure 3(a) (II). Due to the resonant behaviour of $C_{0}$ and $L_{0}$, the current through $S_{3}$ is transferred to $S_{2}$. As a result, $I_{S_{2}}$ approaches $I_{d}$.

As,

$I_{d}=I_{S_{2}}+I_{S_{3}}$

the system, at the end of the transient, is at the condition: $I_{S_{2}}=I_{d}$ and $I_{S_{3}}=0$. Now the current through $S_{3}$ is zero, which allows the controller to switch off $S_{3}$ without any power loss. Mode 2 is operated for a commanded amount of time, $t_{a}$, during which the capacitor $C_{0}$ is reverse charged (as the terminal connected to the drain of IGBT $S_{3}$ is positively charged) to a voltage which is sufficient to forward bias the IGBTs $S_{3}$ and $S_{4}$ in Modes 3 and 4 .

Mode 3

Once there is sufficient voltage bias to turn on $S_{3}$ and $S_{4}$, IGBT $S_{3}$ is turned on. This transfers the current from $S_{2}$ to $S_{3}$. At the end of the transient, the DC link is at a state similar to the start of Mode 2, except for the fact that the resonant tank is charged in the opposite direction [Figure 3(a)(III)] such that $S_{4}$ can be switched on in the next step. Similar to previous modes, $S_{2}$ can be switched off now without any switching loss.

\section{Mode 4}

The operation of mode 4 is depicted in Figure 3(a)(IV). Mode 4 is started by firing $S_{4}$ on. The voltage demanded for this operation is provided by $C_{0}$, which is charged sufficiently during Mode 2. After switching $S_{4}$ on, the current through $S_{1}\left(=I_{m}\right)$ resonantly transfers to $S_{4}$ commencing Mode 4; and when the transfer is completed, $S_{1}$ can be turned off at a power loss of zero. This mode is operated for a time period, $t_{d d}$, during which the capacitor $C_{0}$ is charged (as terminal connected to the drain of the $S_{3}$ IGBT is negatively charged) to a voltage 


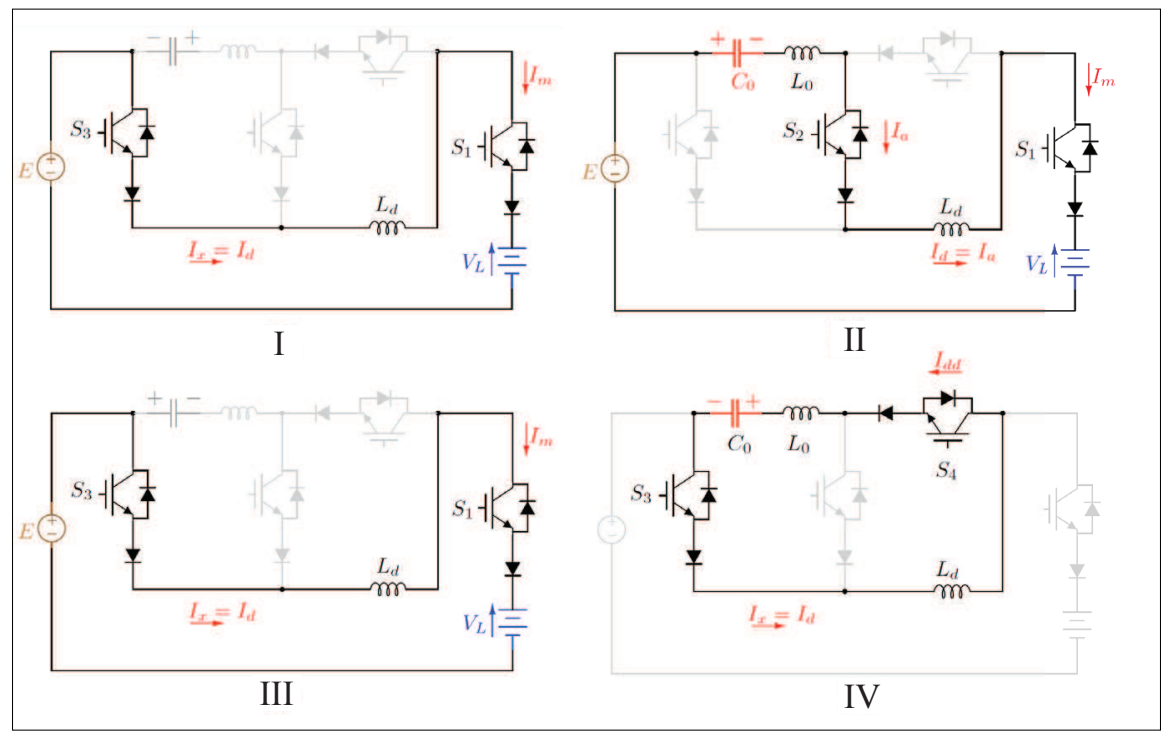

Figure 3(a): The four modes of operation of the FCQDL converter

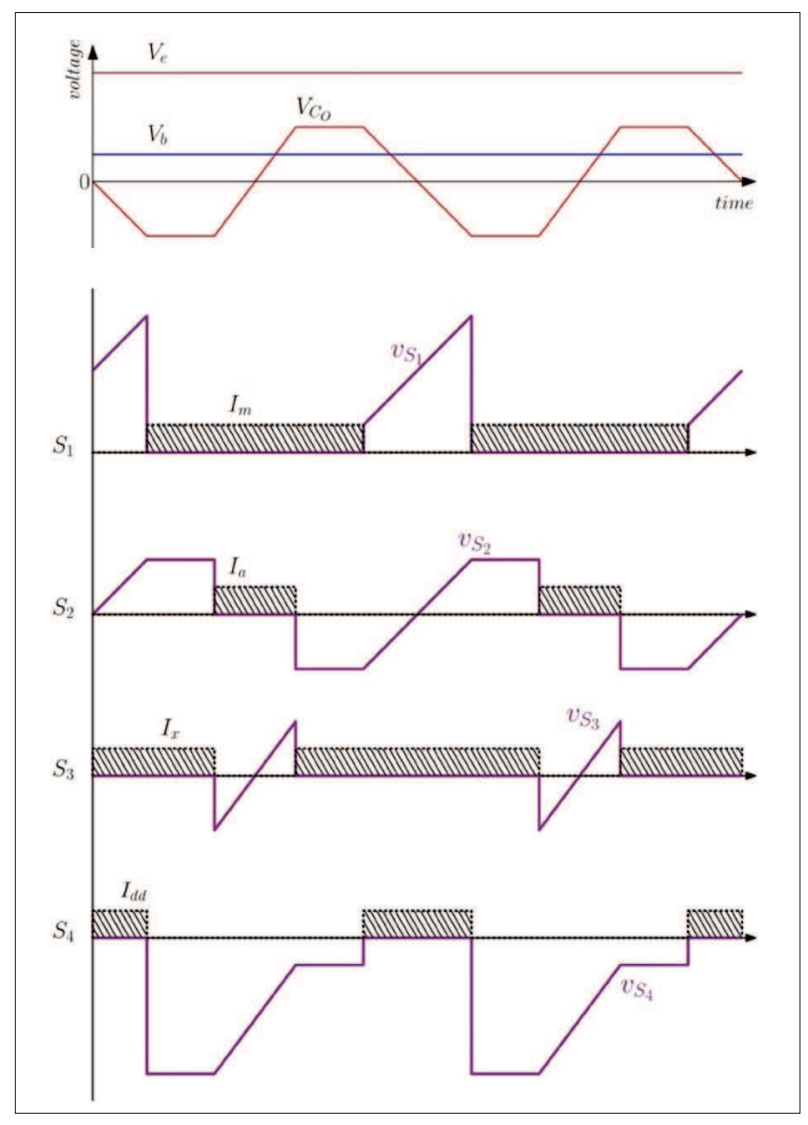

Figure 3(b): Variation of voltages across the four switches $\left(v_{S_{1}}, v_{S_{2}}, v_{S_{3}}, v_{S_{4}}\right)$ and the resonant capacitor for one cycle ( $E$ is input DC voltage, $V_{b}$ is the battery voltage and $V_{C_{O}}$ is the resonant capacitor voltage where $S_{3}$ side is considered as the positive terminal) which is adequate to forward bias $S_{2}$ in the next cycle's Mode 2 operation (Abeyratne \& Aydemir, 1994). When the capacitor, $C_{0}$ is charged to the required level, the next switching cycle is started. Once $S_{1}$ is switched on, the equivalent DC link converter circuit corresponds to the circuit at Mode 1. Therefore, this switching pattern can be continued throughout the whole charging process.

Remark 1

Figure 3(b) shows the voltages across the resonant capacitor and the four switches for a complete switching cycle of the converter. Here, the values chosen for the voltages $V_{c o}, V_{b}$ and $V_{e}$ are for illustrative purposes only; however, they provide adequate basis for qualitative analysis. The voltages across the four switches actually represent the voltage across each IGBT + diode combination where the on-state voltage across the combination is assumed to be zero in contrast to off-state voltage levels. The derivation of voltage waveforms are achieved using simple application of KVL, assuming $I_{d}$ is constant. However, the point at which the voltage transient occurs has a slight offset in the time axis when the soft switching approach is applied to the current waveforms (here the soft switching edges are not shown in current waveforms as we are interested in the overall variation of the voltage across the devices).

Remark 2

$S_{1}$ is turned on throughout modes 1, 2 and 3 . Hence, the width of the current pulse, $t_{m}$, is determined upon the time spent on the first three modes. 


\section{Remark 3}

The minimum value of $t_{d d}$ depends on the time consumed by $C_{0}$ to be charged enough to forward bias $S_{3}$. The time $t_{d d}$ allows the chemical reactions inside the battery to settle down before receiving the next current pulse, hence avoiding unwanted chemical reactions and heat generation. The overall power transfer to the battery is improved as a result.

2) Thyristor bridge: The thyristor bridge acts as the controlled voltage source to the FCQDL converter. The thyristor bridge takes the boost converter output voltage as its input, and delivers a voltage value of $V_{e}$ to the DC link converter by strategically switching the thyristors. Suppose the current required to charge the battery is denoted by $I_{d_{r e f}}$; based on the current through inductor $L_{d}, I_{d}$, the output of the thyristor bridge is decided as follows:

$$
V_{e}=\left\{\begin{array}{c}
V_{d c}, I_{d}<I_{d_{r e f}} \\
0, I_{d}>I_{d_{r e f}}
\end{array}\right.
$$

The need for a controlled voltage output, which switches between $V_{d c}$ and 0 is mainly to control the inductor $L_{d}$ that carries current throughout all the modes of operations of the DC link converter. As discussed previously, the current $I_{d}$ through inductor $L_{d}$ can be assumed constant for a few switching cycles. However, as time goes on, the change in $I_{d}$ would become more noticeable for an input of $V_{d c}$ and would result in the condition $I_{d}>I_{d_{\text {ref }}}$. Once this condition is detected, the thyristor bridge output is set to $0 \mathrm{~V}$, which will gradually decrease the magnitude of $I_{d}$ back to $I_{d_{r e f}}$ within the next few hundred switching cycles. The toggling between $V_{d c}$ and 0 is repeated every few hundred switching cycles to maintain $I_{d}$ within the bound of the desired current value.

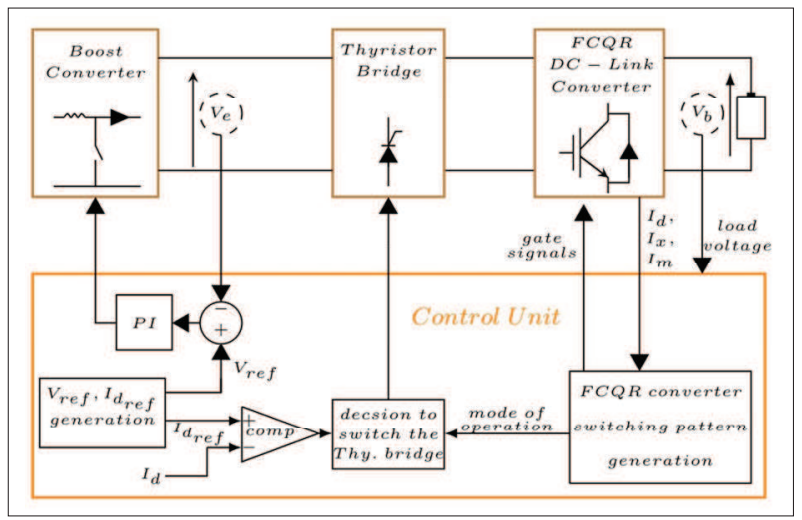

Figure 4: Control of CC phase
It is important to note that switching of the thyristor bridge is done during Mode 4 of the DC link converter, to avoid undesirable charging of the resonant tank.

3) Boost converter: The boost converter generates the voltage $V_{d c}$ required by the DC link converter according to the following equation

$$
V_{d c}=\frac{1}{1-D} V_{i n}
$$

$V_{\text {in }}$ denotes the input voltage to the boost converter and $D$ represents the switching duty cycle of the boost converter. The value of $V_{d c}$ is varied depending on the current consumed by the battery during the CC phase, and is held constant during the CV phase.

\section{Control implementation for $\mathrm{CC}$ and $\mathrm{CV}$ phases}

The overall control implementation of the charger circuit is illustrated in Figure 4. The control implementation for the circuit can be explained as follows.

\section{Constant current (CC) charging phase}

The main aim of the charging circuit in this phase is to continuously provide a current pulse stream of magnitude $I_{d_{\text {ref }}}$ to the high capacity battery. The value of $I_{d_{\text {ref }}}$ is set such that the average value of the current pulse stream transferred into the battery is equal to the recommended charging current, $I_{c}$, of the battery.

The corresponding equation for the charging rate can be represented as,

$$
I_{c}=I_{d_{\text {ref }}} \frac{t_{m}}{T_{p}}
$$

Furthermore, when $I_{d_{r e f}}$ is delivered to the battery, $I_{d}$ equals $I_{d_{r e f}}$ and it is known that $I_{d}$ can be assumed constant for several switching cycles. Based on this fact, the following relationship can be identified.

$$
V_{r e f}=V_{b}+I_{d} \cdot Z
$$

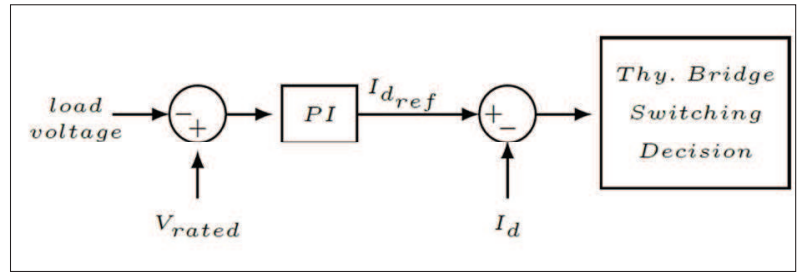

Figure 5: Control of CV phase 
Here, $Z$ represents the total impedance of the DC link converter at a given stage, and $V_{r e f}$ is the reference input for the boost converter controller. Generally, the values for $t_{m}$ and $T_{p}$ are fixed depending on the battery type used. Therefore, according to equation (8), $I_{d_{r e f}}$ is expressed as,

$$
I_{d}=I_{d_{r e f}}=I_{c} \frac{T_{p}}{t_{m}}
$$

Based on equation (9), the value of $V_{\text {ref }}$ is proportionally increased/decreased based on the reading of $I_{d}$ such that $I_{d} \approx I_{d_{\text {ref }}}$ is satisfied. The boost converter output $\left(V_{d c}\right)$ then follows $V_{\text {ref }}$ with the aid of a simple PI controller. Once $I_{d}$ is within a certain vicinity from $I_{d_{\text {ref }}}$, the lower level controller of the thyristor bridge switches the thyristor bridge output between $V_{d c}$ and 0 to maintain $I_{d}$ at desirable levels. Note that the value of $V_{b}$ will increase with time which causes $V_{r e f}$ to increase accordingly. Hence, the controller is actually a PI tracking controller.

Once $V_{b}$ reaches the rated value of the battery, the control methodology switches to the $\mathrm{CV}$ phase.

\section{Constant voltage (CV) charging phase}

In this phase, the voltage across the battery should be kept constant at the rated value specified by the battery manufacturer. Evidently, the average current supplied to the battery should be reduced with time in this phase in order to keep the terminal voltage at a constant value as illustrated in Figure 6. As shown in Figure 4, the terminal voltage of the battery $\left(V_{b}\right)$ is sensed and passed to the control unit.

The control circuit compares the sensed battery terminal voltage with the rated terminal voltage. When the battery terminal voltage exceeds the rated value, the control circuit decreases the magnitude of the $I_{d_{\text {ref }}}$ value by a pre-defined amount. Note that the boost converter output $V_{d c}$ is not further increased in this phase; and therefore, the DC link current $I_{d}$ drops gradually. Consequently, the magnitude of the current pulses fed to the battery is reduced. This loop continues further reducing the value of the DC link current $I_{d}$ throughout the CV phase. This reduces the average current fed to the battery in such a way that the terminal voltage of the battery is not exceeded beyond its rated value.

Note that once the control unit sets a value for $I_{d_{r e f}}$, the lower level thyristor controller switches every few hundred switching cycles to maintain the DC link current around $I_{d_{r e f}}$. The control scheme is shown in Figure 5.

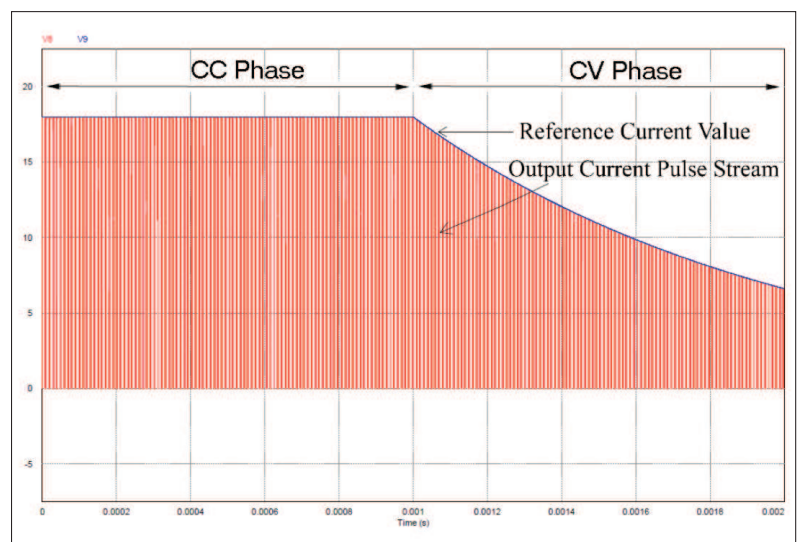

Figure 6: Variation of current pulse stream height with time

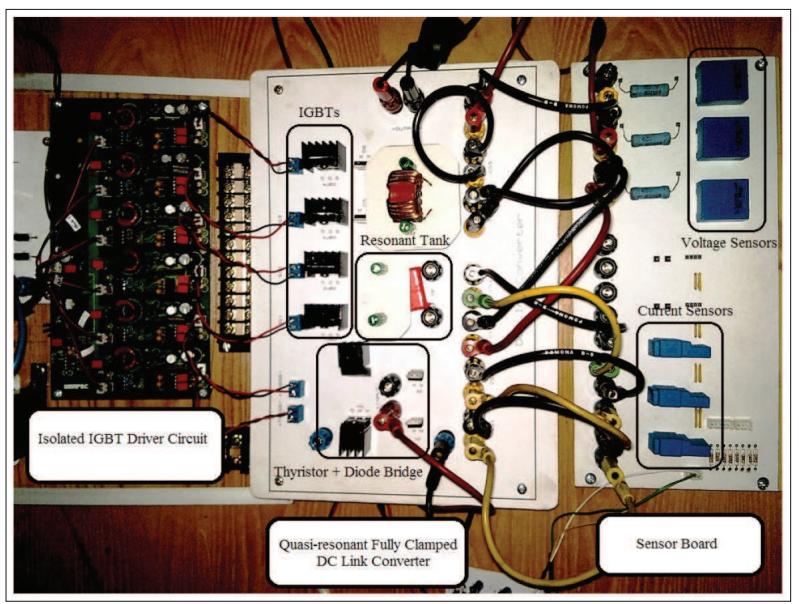

Figure 7: Hardware prototype setup

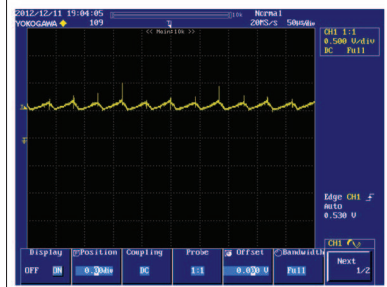

(a)

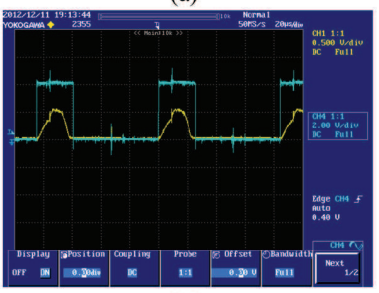

(c)

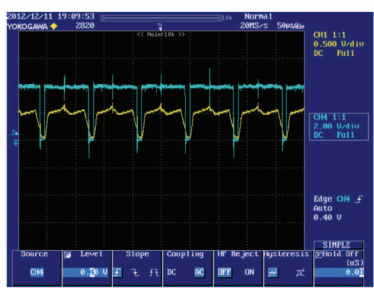

(b)

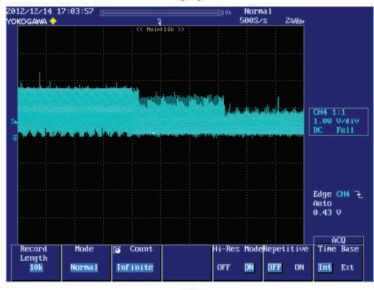

(d)
Figure 8: Experimental measurements of the DC link converter 


\section{SIMULATION AND EXPERIMENTAL RESULTS}

\section{Simulation results}

Simulations were carried out using the PSIM ${ }^{\mathrm{TM}} 9.0$ software to analyse the performance of the proposed charging methodology. The zero-current switching behaviour of these devices is illustrated under the subsection Experimental Results. More results on the simulations are presented in Abeyratne et al. (2012).

The simulation results obtained for the control topology implemented for the $\mathrm{CC}$ and $\mathrm{CV}$ phases are presented in Figure 6. The value of output current pulse magnitude is based on the DC link current $\left(I_{d}\right)$, and follows the reference DC link current value $\left(I_{d_{\text {ref }}}\right)$ as decided by the control unit.

Furthermore, simulations were carried out for a level-2 charger system with $7.2 \mathrm{~kW}$ load power to validate the effects on switching losses due to soft switching of the IGBTs. For this case $I_{d}$ was maintained at $33 \mathrm{~A}$. The proposed topology showed a total switching loss of $0.2 \mathrm{~W}$ for the above system. This agrees with the theory that zero-current switching ideally proposes zero switching losses. However, for the same power and load conditions, the corresponding hard switching scheme (using a single IGBT switch) gives a total switching loss of $19 \mathrm{~W}$. Therefore, the soft switching method offers significantly lower switching losses in contrast to hard switching, hence, results in increased overall system efficiency. For the simulation it was assumed that the turn-on and turn-off losses are equivalent for a switch.

\section{Experimental results}

The pulse charger prototype developed based on the proposed concept is depicted in Figure 7. The prototype consists of an isolated IGBT driver board (left), quasi resonant fully clamped DC link converter (center) and a voltage and current sensor unit (right). For the prototype, the values of the resonant tank components in the DC link converter were, $L_{0}=1 \mathrm{uH}$ and $C_{0}=47 \mathrm{nF}$. The current source inductor value was selected as $L_{\mathrm{d}}=2 \mathrm{mH}$.

1) Experimental waveforms: Figure 8(a) to Figure 8(d) present experimental waveforms defining basic operation of the DC-link and its control. Figure 8(a) shows that the current through $L_{\mathrm{d}}$ is maintained constant around $I_{\mathrm{d}}=0.5 \mathrm{~A}$ for the low-power prototype.
The small ripple is due to the current control function of input thyristor bridge. Figure 8(b) and Figure 8(c) show the load current, $I_{m}$, and the $4^{\text {th }}$ mode circulating current $I_{d d}$, respectively. The corresponding gate signals are also shown (in green colour) and the magnitude of the current pulses agree with the magnitude of $I_{d}$.

Furthermore, Figure 8(d) shows how the load current, $I_{m}$, can be reduced in the $\mathrm{CV}$ phase. Load current through a battery load in the CV phase is continuously commanded to be reduced. This is achieved by changing $I_{d_{r e f}}$ in the control unit. In order to mimic the situation in a faster dynamic condition for the sake of illustration, here, $I_{d_{r e f}}$ is manually commanded to be reduced in two discrete steps. Consequently, the load current magnitude is reduced accordingly as shown in Figure 8(d).

The motive behind the operation of a zero-currentswitching scheme, as implemented in the work is identified at two stages: 1) at turn-on, the current through the switch is slowly increased towards the programmed value, over which the device voltage is constrained to be at a lower value (almost at zero volt); 2) at turn-off, the current of the switching device naturally goes to zero, upon which the switching device turns-off by regaining its blocking capability.

It is to be noted that conduction losses are not dominant compared to the switching losses at the frequencies well above $50 \mathrm{kHz}$. However, the resistance of the circuit (winding, device and copper path) damps the resonant circuit. As a result, the zero current turn-off may fail unless $d i / d t$ of a device at turn-on, is given a minimum bound which becomes a design parameter.

2) Preliminary pulse charging experiments: Preliminary experiments were carried out using a $6 \mathrm{~V}, 4.5 \mathrm{Ah} \mathrm{Pb}$-acid battery to observe the effects on the charging/discharging times of the $\mathrm{Pb}$-acid battery under different methods of charging.

Initially the battery was charged using the conventional CC-CV methodology. The charging curve is illustrated in Figure 9(a). In the CC phase, a current of $400 \mathrm{~mA}$ was continuously fed to the battery until its open circuit voltage reached its rated value of $7.3 \mathrm{~V}$. Then the open circuit terminal voltage of the battery was maintained at $7.3 \mathrm{~V}$ until the average current drawn by the battery dropped to $2 \%$ of its rated value. According to the results obtained, although the battery reaches its rated voltage around 70 minutes, the $\mathrm{CV}$ phase exists for approximately 144 minutes, taking the overall charging time to 214 minutes. 


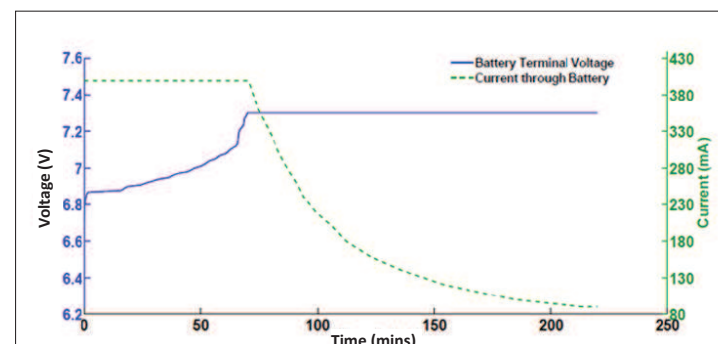

(a)

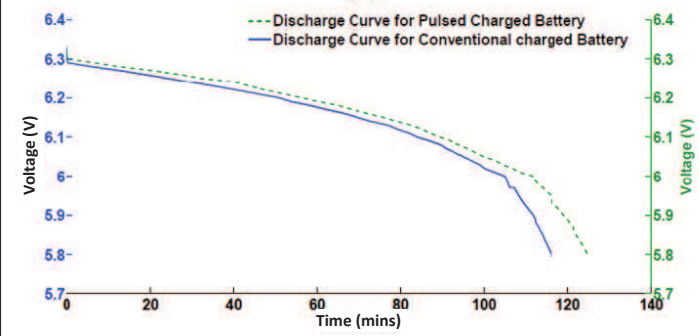

(c)

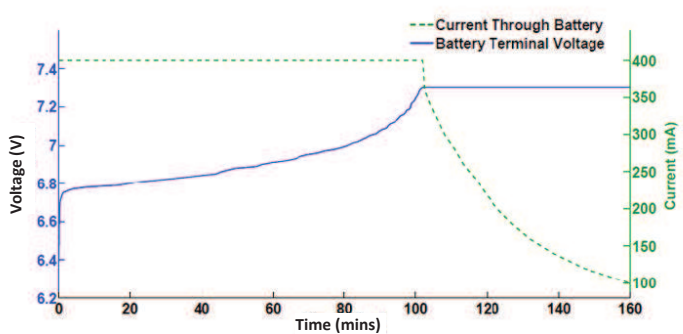

(b)

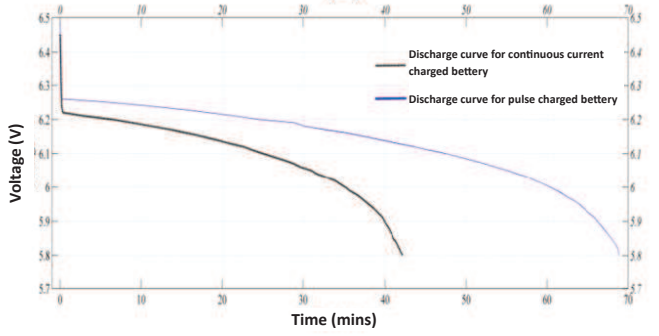

(d)

Figure 9: Experimental charging/discharging comparison between conventional and pulse charging methods

In order to observe the effects of pulse charging, the battery was charged from the same initial state using a current pulse stream with a frequency of $5 \mathrm{kHz}$ (with a $50 \%$ duty ratio). The results showed that the time consumed for constant voltage charging has drastically reduced in this case, reaching the fully-charged state under 185 minutes as shown in Figure 9(b).

It should be emphasised that, in the pulse charging methodology discussed in this paper for both $\mathrm{CC}$ and $\mathrm{CV}$ phases, the current supplied to the battery is in the form of pulses. Experiments carried out using $\mathrm{Pb}$-acid batteries showed that the proposed methodology reduces the charging time by $14.01 \%$.

Progressing upon the results, next, the discharging curves of the battery for the two types of charging methodologies were also obtained and compared to observe the performance of the two cases with regard to their effective charge content. Evidently, the obtained results, as illustrated in Figure 9(c) showed that the pulse charged battery has a better discharging behaviour in contrast to the conventionally charged battery.

An interesting fact that was noted during the experiment was that the time consumed by the $\mathrm{Pb}$-acid battery to reach its rated voltage was lesser when the conventional charging methodology was employed to charge the battery. However, the comparison of retainable charge stored for the two charging methodologies for the $\mathrm{CC}$ phase shows that current pulse charging stores $54.5 \%$ more charge, in the $\mathrm{CC}$ phase with regard to the conventional charging methodology. These results were obtained by ceasing charging of the battery when its terminal voltage reached its rated value, and then by discharging the battery using same conditions for the two methods. The results are shown in Figure 9(d).

\section{CONCLUSION}

Fast and efficient charging of batteries used in automotive (hybrids and EVs) and renewable energy applications is of utmost importance. The charging methodology proposed in this paper exploits a current pulse stream generated with the aid of the fully clamped quasi-resonant DC link converter, which enables zero-current switching through the semiconductor devices. Current pulse charging holds several advantages over the conventional $\mathrm{CC}-\mathrm{CV}$ charging process. While reducing the overall charging time, employing current pulses improves the lifespan, as well as the performance of $\mathrm{Pb}$-acid and other batteries with similar chemical characteristics. The results obtained from the experiments carried out showed that the charging time can be improved by $14 \%$ by replacing the conventional charging mechanism with the pulse charging methodology. The pulse charging also yielded improved discharging curves suggesting that pulse charging improves the lifespan of $\mathrm{Pb}$-acid and $\mathrm{Li}$ ion batteries. 
The comparison of retainable charge stored by the two charging methodologies for the constant current phase shows an interesting result that pulse charging stores $54.5 \%$ more charge in the constant current phase, in contrast to the conventional charging methodology. This makes the constant voltage phase much shorter, dominating the overall charging period to be much shorter. The zero-current switching mechanism introduced ensures efficient power conversion from the renewable energy source to the battery bank.

\section{Acknowledgement}

The research group would like to thank the Peradeniya Engineering Faculty Alumni Association (PEFAA) and the National Science Foundation of Sri Lanka (NSF) for funding this research.

\section{REFERENCES}

1. Abeyratne S. \& Aydemir M. (1994). Current clamped, PWM, quasi-resonant DC link series resonant converter. Conference Record of the 1994 IEEE Industry Applications Society Annual Meeting, volume 2, Denver, USA, 2 - 6 October, pp. $820-826$.

2. Abeyratne S.G., Perera P.S.N., Jayakody H.S., Samarakoon S.M.K.B. \& De Bulathge R.R.S. (2012). Soft switching fast charger for batteries used in renewable energy applications and electric vehicles. Proceedings of the $7^{\text {th }}$ IEEE International Conference on Industrial and Information Systems (ICIIS), Chennai, India, 06 - 09 August.

3. Anderson C. \& Anderson J. (2010). Electric and Hybrid Cars: A History. MacFarland \& Company, Inc., North Carolina, USA.

4. Asadi H., Kaboli S.H.A. \& Safari M.J. (2011). A review on Li-ion battery charger techniques and optimize battery charger performance by fuzzy logic. Proceedings of the International Conference on Information and Intelligent Computing, volume 18, Singapore, pp. 9 - 96.

5. Barton J. \& Infield D. (2004). Energy storage and its use with intermittent renewable energy. IEEE Transactions on Energy Conversion 19(2): 441 - 448.

DOI: http://dx.doi.org/10.1109/TEC.2003.822305

6. Belov D. \& Yang M.H. (2008). Investigation of the kinetic mechanism in overcharge process for Li-ion battery. Solid State Ionics 179(27-32): 1816 - 1821.

DOI: http://dx.doi.org/10.1016/j.ssi.2008.04.031

7. Chen K.K. (2011). A novel application of zero-currentswitching quasi-resonant buck converter for battery chargers. Mathematical Problems in Engineering 2011: 173459 .

DOI: http://dx.doi.org/10.1155/2011/173459

8. Chen L.R. (2009). Design of duty-varied voltage pulse charger for improving Li-ion battery charging response. IEEE Transactions on Industrial Electronics 56(2): 480 487.
DOI: http://dx.doi.org/10.1109/TIE.2008.2002725

9. Etxeberria A. \& Vechiu I. (2010). Hybrid energy storage systems for renewable energy sources integration in microgrids: a review. IEEE International Power Electronics Conference, 21 - 24 June, Sapporo, Japan, pp. 532 - 537.

DOI: http://dx.doi.org/10.1109/ipecon.2010.5697053

10. Garimella N. \& Nair N.K.C. (2009). Assessment of battery energy storage systems for small scale renewable energy integration. Proceedings of the IEEE Region 10 Conference (TENCON 2009), 23 - 26 January, Singapore.

DOI: http://dx.doi.org/10.1109/tencon.2009.5395831

11. Horie H., Abe T., Kinoshita T. \& Shimoida Y. (2008). A study on an advanced lithium-ion battery system for EVs. World Electric Vehicle Journal 2(2): 113 - 119.

12. Hua G. \& Lee F. (1995). Soft-switching techniques in PWM converters. IEEE Transactions on Industrial Electronics 42(6): $595-603$.

DOI: http://dx.doi.org/10.1109/41.4755000

13. Jiang Z. \& Dougal R. (2004). Synergetic control of power converters for pulse current charging of advanced batteries from a fuel cell power source. IEEE Transactions on Power Electronics 19(4): 1140 - 1150.

DOI: http://dx.doi.org/10.1109/TPEL.2004.830044

14. Ke Y., Chuang Y. \& Huang S. (2007). Application of buck zero-current-switching pulse-width-modulated converter in battery chargers. Proceedings of the Industrial and Commercial Power Systems Technical Conference (ICPS 2007), 6 - 11 May, Edmonton, Atlanta.

DOI: http://dx.doi.org/10.1109/icps.2007.4292103

15. Kuperman A., Levy U. \& Goren J. (2011). Modeling and control of the PFC stage for a 50KW EV fast battery charger. Proceedings of the World Congress on Engineering, volume 2, London, UK, pp. 5 - 9.

16. Kutkut N., Wiegman H., Deepak M. \& Novotny D. (1998). Charge equalization for an electric vehicle battery system. IEEE Transactions on Aero Space and Electronic Systems 34(1): $235-246$.

DOI: http://dx.doi.org/10.1109/7.640281

17. Lachs W. \& Sutanto D. (1992). Battery storage plant within large load centres. IEEE Transactions on Power Systems 7(2): $762-769$.

DOI: http://dx.doi.org/10.1109/59.141783

18. Li J., Murphy E., Winnick J. \& Kohl P.A. (2001). The effects of pulse charging on cycling characteristics of commercial lithium-ion batteries. Journal of Power Sources 102(1 - 2): $302-309$.

DOI: http://dx.doi.org/10.1016/S0378-7753(01)00820-5

19. Lin C. \& Yen S. (2008). The application of pulse charge for secondary lithium battery. ECS Transactions 11(29): $55-62$.

DOI: http://dx.doi.org/10.1149/1.2938907

20. Methekar R. \& Ramadesigan V. (2010). Optimum charging profile for lithium-ion batteries to maximize energy storage and utilization. ECS Transactions 25(35): 139 - 146. DOI: http://dx.doi.org/10.1149/1.3414012

21. Mi C. \& Bai H. (2011). Transients of Modern Power Electronic, chapter 1. John Wiley and Sons Inc., New Jersey, USA. 
22. Mi C., Bai H., Wang C. \& Gargies S. (2008). Operation, design and control of dual H-bridge based isolated bidirectional DC-DC converter. IET Power Electronics 1(4): $507-517$.

DOI: http://dx.doi.org/10.1049/iet-pel:20080004

23. Mohan N., Undeland T. \& Robbins W. (2003). Power Electronics: Converters, Applications and Design. John Wiley and Sons Inc., New Jersey, USA.

24. Muthuramalingam A. \& Himavathi S. (2009). Evaluation of power factor corrected AC-DC converters and controllers to meet UPS performance index. International Journal of Electrical and Information Engineering 3(4): 860 - 868. 25. Zhang W., Dong D., Cvetkovic I., Lee F.C. \& Boroyevich D. (2011). Lithium-based energy storage management for DC distributed renewable energy system. Proceedings of the IEEE Energy Conversion Congress and Exposition, 17 - 22 September, Arizona, USA, pp. 3270 - 3277. DOI: http://dx.doi.org/10.1109/ecce.2011.6064210 